Revista de Psicología y Educación / Journal of Psychology and Education, 2020, 15(1), 67-76 (www.rpye.es) Doi: https://doi.org/10.23923/rpye2020.01.186

ISSN: $1699-9517$

\title{
Sentir ilusión por el trabajo docente: inteligencia emocional y el papel del afrontamiento resiliente en un estudio con profesorado de secundaria
}

\author{
Sergio Mérida-López ${ }^{1}$ Natalio Extremera ${ }^{1 *}$, Cirenia Quintana-Orts ${ }^{2}$ y Lourdes Rey \\ 'Universidad de Málaga \\ ¿Universidad de Huelva
}

\begin{abstract}
Resumen: El propósito de esta investigación es analizar las asociaciones entre inteligencia emocional, afrontamiento resiliente y dimensiones de engagement (vigor, dedicación y absorción) en una muestra de 312 docentes de educación secundaria. Además, se examina el papel mediador del afrontamiento resiliente en la relación entre inteligencia emocional y dimensiones de engagement. Los resultados apoyaron las hipótesis propuestas sobre el papel de la inteligencia emocional y el afrontamiento en la predicción de los niveles de engagement del profesorado. Estos hallazgos apuntan a la necesidad de incluir el desarrollo de las habilidades emocionales y el afrontamiento resiliente en la formación del profesorado.

Palabras clave: Inteligencia emocional; Engagement; Afrontamiento resiliente; Docentes
\end{abstract}

To feel engaged with teaching: Emotional intelligence and the role of resilient coping in a study with secondary teachers

\begin{abstract}
This research aims to analyze the associations among emotional intelligence, resilient coping and engagement dimensions (vigor, dedication and absorption) in a sample of 312 secondary school teachers. In addition, the mediator role of resilient coping in the relation between emotional intelligence and engagement dimensions is examined. Results supported the proposed hypotheses about the role of emotional intelligence and resilient coping in predicting teacher engagement. These findings point out the necessity of including the development of emotional skills and resilient coping in teacher training.

Keywords: Emotional intelligence; Engagement; Resilient coping; Teachers.
\end{abstract}

Existe consenso por parte de investigadores e instituciones educativas acerca de señalar la docencia como una ocupación profesional de alto riesgo psicosocial (Extremera, Rey y Pena, 2010; Instituto Nacional de Seguridad e Higiene en el Trabajo, 2015). Además de estudios internacionales que han señalado el impacto negativo de la labor docente sobre el bienestar personal y profesional de este colectivo profesional (Schonfeld, Bianchi y Luehring-Jones, 2017), distintas investigaciones con muestras españolas relacionan la práctica profesional en

Recibido: 28/04/2019 - Aceptado: 24/09/2019 - Avance online: 31/10/2019

*Correspondencia: Natalio Extremera

Universidad de Málaga

C.P: 29071, Málaga, España.

E-mail: nextremera@uma.es

Mérida-López, S., Extremera, N., Quintana-Orts, C. y Rey., L. (Avance Online). Sentir ilusión secundaria con la aparición de problemas físicos y psicosociales (Extremera et al., 2010).

Entre las principales fuentes de estrés del profesorado de secundaria se encuentra el trato con alumnos y otros usuarios del contexto educativo (INSHT, 2015; Travers, 2017). Además, existen múltiples estresores de rol y numerosas demandas emocionales que el profesorado debe afrontar en su trabajo cotidiano y que deterioran su salud y bienestar psicológico (Mérida-López, Extremera y Rey, 2017; Schonfeld et al., 2017). En este sentido, recientes informes como el publicado por el Defensor del Profesor de ANPE Sindicato Independiente han alertado del aumento en la demanda de atención psicológica por parte de los profesionales docentes (ANPE, 2017). Asimismo, entre las consecuencias más 
habituales del estrés docente se encuentran el distanciamiento, la desmotivación y la falta de realización personal y profesional (Travers, 2017).

Las consecuencias del estrés docente tienen un impacto económico, educativo y social que se relacionan con el deterioro de la salud y el bienestar del profesorado (Travers, 2017; Watlington, Shockley, Guglielmino y Felsher, 2010). Sin embargo, no todos los docentes desarrollan problemáticas psicosociales en su contexto profesional. Desde la Psicología Organizacional Positiva, se viene poniendo énfasis en los recursos personales del trabajador, sus antecedentes y sus consecuencias positivas (Salanova, Martínez y Llorens, 2005). El interés de esta nueva aproximación conceptual se centra en analizar las características de aquellas personas y grupos de trabajo que desarrollan un funcionamiento individual y colectivo óptimo en las organizaciones. De acuerdo con este planteamiento, se parte de la base de que el profesional posee una serie de fortalezas, virtudes y aspectos positivos que le permiten alcanzar un "funcionamiento óptimo" en su organización (Salanova et al., 2005). Desde esta línea, se ha otorgado un papel destacado al engagement. Este constructo es entendido como un estado motivacional positivo y persistente relacionado con el trabajo, integrado por las dimensiones de vigor, dedicación y absorción (Schaufeli, Salanova, González-Romá y Bakker, 2002). El engagement constituye un factor clave en la promoción de la salud y el bienestar de los profesionales docentes debido a sus efectos sobre el bienestar psicológico, la satisfacción laboral, el rendimiento o los comportamientos prosociales en el trabajo (Taris, Leisink y Schaufeli, 2017).

Con respecto a las principales causas del engagement, encontramos factores organizacionales (autonomía, apoyo de compañeros y superiores, entre otros) y también factores personales, incluyendo variables sociodemográficas, actitudinales y/o recursos personales (Bakker, Demerouti y Sanz-Vergel, 2014). Entre estos recursos, la inteligencia emocional se ha convertido en foco de atención de diversos investigadores en las últimas décadas (Akhtar, Boustani, Tsivrikos y Chamorro-Premuzic, 2015; Extremera, Mérida-López, Sánchez-Álvarez y Quintana-Orts, 2018).

El constructo de inteligencia emocional engloba diferencias individuales en las habilidades de percibir, utilizar, comprender y regular las propias emociones y las de otros (Mayer y Salovey, 1997). Dicho constructo ha experimentado una creciente importancia en el contexto educativo a lo largo de los últimos años. Como prueba de ello, se encuentran numerosas investigaciones que avalan los beneficios de la inteligencia emocional en el contexto docente (Extremera y FernándezBerrocal, 2015; Mérida-López y Extremera, 2017). Por ejemplo, un estudio reciente con profesorado de secundaria ha mostrado una relación significativa entre los niveles de inteligencia emocional de los participantes y sus niveles de desgaste profesional y desajuste psicológico (Martínez-Monteagudo, Inglés, Granados, Aparisi y García-Fernández, 2019). Además, la inteligencia emocional ha sido considerado un recurso personal clave que ayuda a mejorar el bienestar y el desempeño docente (Vesely, Saklofske y Leschied, 2013).

A pesar de la evidencia acumulada en los últimos años que relaciona la inteligencia emocional con mayores niveles de vigor, dedicación y absorción en el trabajo docente (Durán, Extremera y Rey, 2010; Mérida-López, Bakker y Extremera, 2019), son escasos los estudios que examinan los posibles mecanismos explicativos en la relación entre inteligencia emocional y engagement docente. La literatura actual sugiere que una posible variable que podría explicar por qué los docentes con más inteligencia emocional informan de mayores niveles de estados de ánimo positivos en su trabajo sería el estilo de afrontamiento que utilizan (Bermejo-Toro, Prieto-Ursúa y Hernández, 2016; Zeidner y Matthews, 2018). De hecho, el afrontamiento resiliente ha mostrado ser un recurso de los docentes con influencia sobre sus niveles de desgaste profesional o de engagement (Parker, Martin, Colmar y Liem, 2012). De esta manera, los docentes con mayor habilidad para percibir, 
comprender y manejar sus emociones podrían llevar a cabo un afrontamiento mucho más eficaz ante los estresores docentes habituales (conductas disruptivas de alumnos, conflicto con compañeros...), basado en la creatividad, la tenacidad y el crecimiento personal tras estas dificultades habituales entre docentes (Mansfield, Beltman, Broadley y WeatherbyFell, 2016). Este tipo de afrontamiento podría tener un efecto positivo sobre sus niveles de ilusión en el trabajo (engagement).

A pesar de que tanto la inteligencia emocional como el afrontamiento resiliente se han sugerido como recursos personales de relevancia para el bienestar y el compromiso de los docentes (Mansfield et al., 2016; Vesely et al., 2013), no existen estudios hasta la fecha que hayan relacionado la inteligencia emocional y el afrontamiento resiliente con el engagement docente. Por ello, el objetivo del presente estudio versa sobre el análisis de la relación entre inteligencia emocional, afrontamiento resiliente y dimensiones de engagement (vigor, dedicación y absorción) en una muestra de docentes de secundaria. Además, se pretende examinar el posible papel explicativo del afrontamiento resiliente entre inteligencia emocional y mayores niveles de vigor, dedicación y absorción en el trabajo. Siguiendo la literatura previa, se plantean las siguientes hipótesis:

Primero, aquellos docentes con mayores puntuaciones de inteligencia emocional mostrarán un mayor afrontamiento resiliente y mayores niveles de engagement en su trabajo. Asimismo, aquellos docentes con mayor uso de estrategias de afrontamiento resiliente informarán de mayores niveles

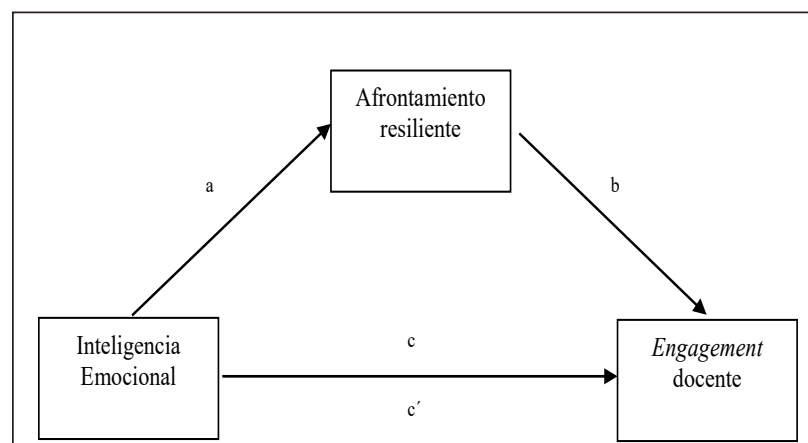

Figura 1: Modelo de mediación propuesto con inteligencia emocional, afrontamiento resiliente y engagement docente. de engagement. Finalmente, siguiendo el modelo de mediación propuesto (ver Figura $1)$, aquellos docentes con mayores niveles de inteligencia emocional llevarán a cabo un afrontamiento más resiliente de los estresores docentes diarios, lo que se relacionará a su vez de forma positiva con las dimensiones de engagement.

\section{MÉTODO}

\section{PARTICIPANTES}

La muestra está compuesta por un total de 312 profesionales docentes, de los cuales 148 pertenecen al sexo masculino $(47,4 \%)$ y 164 al femenino $(52,6 \%)$. Los participantes desarrollaban su labor profesional en distintos centros de educación secundaria de la provincia de Málaga. La media de edad fue de 45,22 años (DT = 8,73; Rango $=26$ 67 años). El estado civil de los participantes fue soltero/a $(n=80 ; 25,6 \%)$, casado/a $(n=$ $201 ; 64,4 \%)$, separado/a o divorciado/a $(n=$ $18 ; 5,8 \%)$, viudo/a $(n=1 ; 0,3 \%)$ y pareja de hecho $(n=5 ; 1,6 \%)$. Siete participantes no informaron de su estado civil. Finalmente, los participantes informaron de una media de 17 años en el cuerpo docente $(D T=9,95)$, con un rango que oscilaba entre un mes y 40 años. Finalmente, la antigüedad media en el centro educativo fue de 9 años $(D T=8,14)$, con un rango que oscilaba entre un mes y 34 años.

\section{INSTRUMENTOS DE EVALUACIÓN}

Además de evaluar variables sociodemográficas como la edad, el género, el estado civil, la antigüedad en el cuerpo docente o la antigüedad en el centro educativo actual, se utilizaron tres cuestionarios autoinformados.

Para evaluar los niveles de inteligencia emocional se utilizó la versión en castellano del cuestionario Wong and Law Emotional Intelligence Scale (WLEIS; original de Wong y Law, 2002). Este instrumento se compone de 16 ítems que evalúan el nivel de inteligencia emocional auto-percibido con una escala tipo Likert de 7 puntos que oscila 
desde "Completamente en desacuerdo" (1) hasta "Completamente de acuerdo" (7). El instrumento evalúa cuatro dimensiones: evaluación de las propias emociones o percepción intrapersonal (4 ítems) $(\alpha=, 77)$ ("Siempre sé si estoy o no estoy feliz"); evaluación de las emociones de los demás o percepción interpersonal (4 ítems) $(\alpha=, 78)$ ("Conozco siempre las emociones de mis amigos a través de sus comportamientos"); Uso de las emociones o asimilación emocional (4 ítems) $(\alpha=$,79) ("Siempre me fijo metas y luego intento hacerlo lo mejor para alcanzarlas") y regulación de las emociones (4 ítems) $(\alpha=, 83)$ ("Soy capaz de controlar mis propias emociones"). No obstante, en este trabajo se empleó la puntuación total con el propósito de evaluar la inteligencia emocional de manera global. La versión en castellano ha mostrado adecuadas propiedades psicométricas en muestras españolas (Extremera, Rey y Sánchez-Álvarez, 2019). La fiabilidad para la puntuación total fue excelente $(\alpha=, 90)$.

La evaluación del afrontamiento resiliente se llevó a cabo con la adaptación al castellano de la escala breve de estrategias resilientes (Brief Resilient Coping Scale, BRCS; Limonero et al., 2010; original de Sinclair y Wallston, 2004). Este instrumento se compone de cuatro ítems con una escala tipo Likert de 5 puntos que oscilan desde "No me describe en absoluto" (1) hasta "Me describe muy bien" (5). Con esta escala se valora la frecuencia de la estrategia de afrontamiento descrita ("Busco formas creativas para cambiar las situaciones difíciles"). La versión adaptada al castellano muestra adecuadas propiedades psicométricas en muestras españolas (Limonero et al., 2010). En este estudio, el índice de fiabilidad fue bueno $(\alpha=, 67)$.

La evaluación del engagement se realizó con la adaptación al castellano de la versión de 15 ítems del cuestionario Utrecht Work Engagement Scale (UWES-15, Schaufeli et al., 2002; original de Salanova, Schaufeli, Llorens, Peiró y Grau, 2000). Este instrumento mide 15 ítems con una escala tipo Likert de 7 puntos que oscilan desde "Nunca" (0) hasta "Todos los días" (6). Los ítems del cuestionario evalúan tres dimensiones: vigor (5 ítems) $(\alpha=, 86)$ ("En mi trabajo me siento lleno/a de energía"); dedicación (5 ítems) $(\alpha=, 88)$ ("Estoy entusiasmado/a con mi trabajo") y absorción (5 ítems) $(\alpha=, 88)$ ("El tiempo vuela cuando estoy trabajando").

\section{PROCEDIMIENTO}

La recogida de información fue llevada a cabo mediante técnicas de reclutamiento a través de estudiantes, las cuales han mostrado su efectividad en estudios previos en el ámbito organizacional (Wheeler, Shanine, Leon y Whitman, 2014). Diversos estudiantes de la Universidad de Málaga fueron instruidos para cumplir con los requerimientos de la investigación (voluntariedad, anonimato y confidencialidad de las pruebas). En este sentido, se garantizó el cumplimiento de la normativa vigente sobre protección de datos puesto que no se requirió ningún tipo de información personal en la cumplimentación de las escalas. Los estudiantes accedieron a distintos centros de secundaria de la provincia de Málaga y repartieron los cuestionarios en formato papel y lápiz a responsables de los centros, explicando el propósito del estudio. Los participantes fueron informados de que al cumplimentar los cuestionarios y entregarlos estaban dando su consentimiento informado para participar en la investigación. Cuando los cuestionarios se cumplimentaron en un plazo dado de cuatro semanas, los estudiantes recogieron dichos cuestionarios y los devolvieron al equipo investigador. Alrededor de 600 cuestionarios fueron entregados a los centros, resultando una tasa de respuesta del 52\%. El procedimiento del estudio fue aprobado por el Comité Ético de la Universidad de Málaga (PSI2012-38813).

\section{ANÁLISIS DE DATOS}

Respecto al diseño de investigación, el presente trabajo consistió en un estudio correlacional con un diseño transversal. Los datos obtenidos a través de las diferentes escalas utilizadas han sido tratados con el software estadístico SPSS. 24.0. En primer lugar, se analizaron los estadísticos descriptivos 
de la muestra en relación con las variables sociodemográficas recogidas. En segundo lugar, se llevaron a cabo correlaciones de Pearson entre las variables inteligencia emocional, afrontamiento resiliente y dimensiones de engagement. Finalmente, se analizaron tres modelos de mediación para comprobar si el afrontamiento resiliente actuaba como mecanismo mediador en la relación de la inteligencia emocional con vigor, dedicación y absorción. Para los análisis de mediación se empleó la macro PROCESS de Hayes (2013).

\section{RESULTADOS}

\section{ANÁLISIS DESCRIPTIVOS Y DE CORRELACIÓN}

Como se puede observar en la Tabla 1, el análisis de correlación encontró que la variable inteligencia emocional se asociaba positivamente con el afrontamiento resiliente y con las dimensiones de engagement docente. Asimismo, se encontró que el afrontamiento resiliente se asociaba positivamente con las dimensiones de engagement docente.

Tabla 1

Estadísticos descriptivos y correlaciones de las variables objeto de estudio

\begin{tabular}{|l|c|c|c|c|c|}
\hline & 1 & 2 & 3 & 4 & 5 \\
\hline $\begin{array}{l}\text { 1. Inteligencia } \\
\text { emocional }\end{array}$ & - & & & & \\
\hline $\begin{array}{l}\text { 2. Afrontamiento } \\
\text { resiliente }\end{array}$ &, $58^{\star \star}$ & - & & & \\
\hline 3. Vigor &, $43^{\star *}$ &, $35^{\star *}$ & - & & \\
\hline 4. Dedicación &, $47^{\star *}$ &, $41^{* *}$ &, $78^{\star *}$ & - & \\
\hline 5. Absorción &, $31^{\star *}$ &, $28^{* *}$ &, $64 * *$ &, $69 * *$ & - \\
\hline Media & 5,48 & 3,97 & 5,05 & 5,08 & 4,78 \\
\hline DT & 0,70 & 0,56 & 0,85 & 0,98 & 1,08 \\
\hline Mín. & 2,50 & 2 & 1,40 &, 40 & 0 \\
\hline Máx. & 6,94 & 5 & 6 & 6 & 6 \\
\hline Nota: ** $<<, 01$ & & & & \\
\hline
\end{tabular}

\section{ANÁLISIS DE MEDIACIÓN}

Se llevaron a cabo tres análisis de mediación independientes para cada una de las dimensiones de engagement docente (vigor, dedicación y absorción). En la Tabla 2 se muestran los resultados principales del análisis de mediación. Los hallazgos indican que la inteligencia emocional se asocia positivamente con el afrontamiento resiliente, que a su vez se relaciona positivamente con las dimensiones de engagement docente. Los resultados muestran que la relación directa entre inteligencia emocional y las dimensiones de engagement docente continúa siendo significativa una vez se ha incluido en el modelo la variable de afrontamiento resiliente. Esto indica que el afrontamiento resiliente es un mediador parcial en la relación entre inteligencia emocional y las tres dimensiones de engagement docente. Así, estos resultados sugieren que el vínculo entre inteligencia emocional y engagement docente no es enteramente directo, sino que pasa a través del uso de mayores estrategias resilientes que, a su vez, incrementa los niveles de engagement de los docentes.

\section{DISCUSIÓN}

El objetivo del presente estudio fue analizar las asociaciones entre inteligencia emocional, afrontamiento resiliente y engagement en una muestra de profesorado de secundaria, así como un modelo de mediación. Los hallazgos encontrados apoyaron las hipótesis propuestas para el presente trabajo. En cuanto al análisis de correlación, se encontró un patrón similar al hallado en estudios previos (Limonero et al., 2010; Mérida-López et al., 2019). Además, los resultados de los análisis de mediación mostraron que el afrontamiento resiliente ejercía como mediador que explicaba la relación de inteligencia emocional con vigor, dedicación y absorción.

Estos hallazgos sugieren que, además de existir una relación directa entre inteligencia emocional y engagement docente, el afrontamiento resiliente podría ser una estrategia adicional que emplearían los 
Tabla 2

Resultados del análisis de mediación para las dimensiones de engagement

\begin{tabular}{|c|c|c|c|c|c|c|c|}
\hline \multirow[b]{2}{*}{ Pasos } & \multicolumn{3}{|c|}{ Modelo de efecto total } & \multicolumn{4}{|c|}{ Modelo de efecto indirecto } \\
\hline & B & SE & $t$ & B & SE & $t$ & IC 95\% \\
\hline Edad $^{a}$ &,- 01 & .01 & $-1,28$ &.,- 01 &, 01 & $-1,02$ & \\
\hline $\mathrm{IE}$ - afrontamiento resiliente $(a)$ & 47 & .04 & $12,60^{\star \star \star}$ & & & & \\
\hline Afrontamiento resiliente - vigor (b) & 21 & .10 & $2,17^{\star}$ & & & & \\
\hline IE - vigor (C) &, 52 & .06 & $8,33^{* \star \star}$ & & & & \\
\hline $\mathrm{IE}-\operatorname{vigor}\left(\mathrm{C}^{\prime}\right)$ & 43 & , 08 & $5,55^{\star \star \star}$ & & & & \\
\hline $\begin{array}{l}\mathrm{IE} \text { - afrontamiento } \\
\text { resiliente - vigor (ab) }\end{array}$ & & & & , 10 &, 05 & & {$[, 01 /, 20]$} \\
\hline $\mathrm{R}^{2}$ & 19 & & & 20 & & & \\
\hline$F(g /)$ & $\begin{array}{l}35,85^{\star \star *} \\
(2,309)\end{array}$ & & & $\begin{array}{l}25,77^{\star \star \star} \\
(3,308)\end{array}$ & & & \\
\hline Edad $^{a}$ &,- 01 & .01 & $-2,00^{*}$ &,- 01 & .01 & $-1,63$ & \\
\hline $\mathrm{IE}$ - afrontamiento resiliente $(a)$ & 47 & .04 & $12,60 * * *$ & & & & \\
\hline Afrontamiento resiliente - dedicación (b) & 34 &, 11 & $3,19 * *$ & & & & \\
\hline IE - dedicación (C) & 66 &, 07 & $9,37 * \star *$ & & & & \\
\hline IE - dedicación (c') &, 50 & .09 & $5,87^{\star * \star}$ & & & & \\
\hline IE - afrontamiento resiliente - dedicación (ab) & & & & 16 & .06 & & {$[, 04 /, 30]$} \\
\hline$R^{2}$ & 23 & & & 26 & & & \\
\hline$F(g /)$ & $\begin{array}{c}46,43^{* * *} \\
(2,309)\end{array}$ & & & $\begin{array}{c}35,25^{\star * *} \\
(3,308)\end{array}$ & & & \\
\hline Edad $^{a}$ &,- 01 & 01 & $-0,95$ &,- 01 & 01 & $-0,69$ & \\
\hline $\mathrm{IE}$ - afrontamiento resiliente $(a)$ & 47 & ,04 & $12,60 * * *$ & & & & \\
\hline Afrontamiento resiliente - absorción (b) & 27 & .13 & $2,15^{\star}$ & & & & \\
\hline IE - absorción (c) & 48 & 08 & $5,70 * * \star$ & & & & \\
\hline IE - absorción (c') & 35 &, 10 & $3,40 * *$ & & & & \\
\hline IE - afrontamiento resiliente - absorción (ab) & & & &,- 28 &, 08 & & {$[-, 46 /-, 15]$} \\
\hline $\mathrm{R}^{2}$ & , 10 & & & , 11 & & & \\
\hline$F(g /)$ & $\begin{array}{l}16,85^{* * *} \\
(2,309)\end{array}$ & & & $\begin{array}{c}12,91^{\star * *} \\
(3,308)\end{array}$ & & & \\
\hline \multicolumn{8}{|c|}{$\begin{array}{l}\text { Nota: } \mathrm{n}=312 \text {. IE = Inteligencia Emocional. } a, b, c \text { y } c^{\prime} \text { representan coeficientes de regresión no estandarizados: a = asociación directa } \\
\text { entre IE y vigor, dedicación, absorción; } b=a \text { asociación directa entre afrontamiento resiliente y vigor, dedicación, absorción; } c=\text { efecto total } \\
\text { entre IE y vigor, dedicación, absorción (sin tener en cuenta la influencia de la variable afrontamiento resiliente); } c^{\prime}=\text { efecto directo entre IE } \\
\text { y vigor, dedicación, absorción (teniendo en cuenta el papel de la variable afrontamiento resiliente); ab = efecto indirecto entre IE y vigor, } \\
\text { dedicación, absorción a través del afrontamiento resiliente. IC } 95 \%=\text { Intervalo de Confianza al } 95 \% \text { con muestreos múltiples. }{ }^{\circ} \text { Edad se } \\
\text { utilizó como variable control. Mediación total }=c \text { se reduce por parte de ab a un efecto no significativo de } c^{\prime} ; \text { mediación parcial = } c \text { se } \\
\text { reduce por la influencia de ab, pero } c^{\prime} \text { permanece con un efecto significativo. }{ }^{*} p<, 05 ;{ }^{* *} p<, 01 ;{ }^{* * *} p<, 001 \text {. }\end{array}$} \\
\hline
\end{tabular}

docentes emocionalmente inteligentes (Zeidner y Matthews, 2018). Así, estos resultados apoyan el argumento de que los docentes con mayor habilidad auto-percibida para percibir, comprender y regular sus estados emocionales y los de su alumnado desarrollan estrategias más resilientes para afrontar los contratiempos y adversidades que pueden surgir en el contexto educativo (Richards, Hemphill y Templin, 2018), utilizando formas creativas, optimistas y de desarrollo personal para afrontarlos, lo cual podría redundar en mayores niveles de ilusión en su trabajo. Por el contrario, es posible que los docentes con bajos niveles de inteligencia emocional no sepan desarrollar estrategias resilientes ante las adversidades educativas a las que se enfrentan diariamente en el aula y, por ello, empiecen a experimentar niveles más bajos de ilusión hacia la enseñanza, menor energía, más baja dedicación hacia sus alumnos y menos momentos de absorción en clase. En esta línea, los programas de formación del profesorado centrados en la 
creación de entornos positivos de aprendizaje podrían incorporar ciertos módulos donde se instruyese al profesorado a identificar conflictos y contratiempos diarios, ayudarles a reconocer las emociones negativas y cómo afectan a su rendimiento y al clima del aula $y$, finalmente, enseñarles formas eficaces de regular las emociones que originan estas adversidades. Así, se sentirían más confiados para poner en práctica estrategias regulativas y resilientes en su ejercicio profesional diario, fortaleciendo su percepción de que su trabajo es retador y desafiante y, por ende, incrementando sus niveles de ilusión docente.

El presente trabajo presenta algunas limitaciones que deberían tenerse en cuenta en el desarrollo de futuras aportaciones en esta línea. El uso de pruebas autoinformadas podría complementarse en futuros estudios con entrevistas o diarios de seguimiento, de manera que se pudiera examinar el impacto particular de estresores diarios y cómo los docentes que afrontan de manera resiliente presentan a lo largo del tiempo mayores niveles de energía y dedicación en su trabajo (Travers, 2017 ). Igualmente, el uso de diseños prospectivos y longitudinales nos permitiría establecer relaciones causales entre las variables examinadas.

Debido a las implicaciones del engagement sobre aspectos de gran impacto para el Sistema Educativo como el absentismo, las rotaciones o el abandono de la carrera docente (Mansfield et al., 2016; Taris et al., 2017), sería conveniente que en futuras investigaciones se analice el papel de los recursos personales positivos - en este caso la inteligencia emocional y el afrontamiento resiliente - en relación con variables como la intención de abandono de la enseñanza. Finalmente, los profesores noveles constituirían un colectivo de gran interés debido a la oportunidad de comprobar si los niveles de inteligencia emocional y afrontamiento resiliente en las primeras etapas de la carrera docente pueden tener relación con el bienestar ocupacional o la intención de abandono de la enseñanza.

A pesar de las limitaciones señaladas, los resultados han mostrado que los docentes más emocionalmente inteligentes informan de un mayor uso de estrategias de afrontamiento resiliente del estrés, mostrando así mayores niveles de ilusión en su trabajo. Por ello, estos hallazgos contribuyen al desarrollo de modelos de bienestar docente más integradores en los que se analicen los recursos personales positivos de los profesionales (Bermejo-Toro et al., 2016; Vesely et al., 2013).

Tal y como sugiere la literatura actual, estos resultados podrían tener implicaciones prácticas para la mejora del ajuste y el bienestar de los futuros docentes. En los últimos años se han realizado aportaciones preventivas para complementar la formación del profesorado de secundaria con herramientas socioemocionales para la mejora de su bienestar personal y ocupacional (Palomera, Briones, Gómez-Linares y Vera, 2017; Vesely, Saklofske y Nordstokke, 2014). Estos datos preliminares podrían contribuir al desarrollo de actividades específicas en futuros programas de entrenamiento en inteligencia emocional con docentes en formación inicial y en activo en los cuales se trabaje el uso de estrategias de afrontamiento resiliente frente a las situaciones adversas cotidianas. Esta formación podría incidir en la mejora de las habilidades emocionales de los docentes y favorecer el uso de estrategias de afrontamiento de tipo resiliente, con lo que se podría reducir el deterioro producido por el estrés en la enseñanza (Mansfield et al., 2016). Asimismo, el uso de estrategias de afrontamiento resiliente podría constituir un predictor de los niveles de motivación y compromiso entre los docentes de secundaria, favoreciendo así su intención de retención (Tait, 2008).

En definitiva, la inteligencia emocional mostró influencia tanto de forma directa como indirecta, a través de las estrategias resilientes, sobre los niveles de engagement docente. Por tanto, el proceso final podría parcialmente depender de los niveles de habilidades emocionales que el profesional posea, lo cual podría aumentar la influencia de la resiliencia sobre la ilusión hacia el trabajo. Estos hallazgos pueden ser relevantes no sólo para desarrollar modelos teóricos que incluyan estas dimensiones y comprender mejor el 
vínculo entre habilidades emocionales e ilusión por el trabajo, sino también para desarrollar programas más efectivos dirigidos al desarrollo de organizaciones positivas y saludables, que incluyan antecedentes distales (incrementar las habilidades emocionales básicas) y proximales (desarrollar estrategias resilientes ante las adversidades) para fomentar la ilusión docente. Los programas dirigidos a la mejora del clima laboral en los centros educativos y a prevenir riesgos psicosociales en la enseñanza podrían incorporar estrategias específicas para incrementar la inteligencia emocional mediante actividades que permitan reconocer las causas de las emociones de frustración, insatisfacción y enfado en la aula, comprender si estas causas son atribuibles a ellos o a otras personas, y, finalmente, tratar de poner en prácticas estrategias de regulación intrapersonal o interpersonal. Con estas propuestas de desarrollo emocional realizadas de forma preventiva, a los docentes se les ayudaría a incrementar su repertorio de estrategias resilientes y de solución de problemas, y, con ello, aumentar sus actitudes laborales positivas y de ilusión por la enseñanza.

En conclusión, estos resultados sugieren la necesidad de atender a la mejora de las habilidades emocionales del profesorado como medio para mejorar los niveles de ilusión docente (Vesely et al., 2013). Asimismo, estos hallazgos sirven como base para incluir el desarrollo de estrategias de afrontamiento resiliente como objetivo específico de los programas de entrenamiento en habilidades emocionales con el profesorado. Esta formación puede favorecer el uso de estrategias más creativas y tenaces para el manejo de los estresores diarios en el contexto docente y, con ello, mejorar los niveles de ilusión por el trabajo.

\section{- Agradecimientos}

Esta investigación ha sido financiada por el proyecto Feder de la Junta de Andalucía (UMA18-FEDERJA-147). Además, esta investigación ha sido realizada bajo el programa de becas FPU (Formación del Profesorado Universitario) financiado por el Ministerio de Educación y Formación Profesional del gobierno de España, concedida al primer autor, Sergio Mérida-López, con referencia FPU16/02238.
- Conflicto de intereses.

Los autores declaran no tener ningún conflicto de intereses.

\section{REFERENCIAS}

Akhtar, R., Boustani, L., Tsivrikos., D. y Chamorro-Premuzic, T. (2015). The engageable personality: Personality and trait El as predictors of work engagement. Personality and Individual Differences, 73, 44-49.

ANPE (2017). Memoria del defensor del profesor (curso 2016-2017). Último acceso el 04 de septiembre de 2018. Disponible en: https:// eldefensordelprofesor.es/openFile. php?link=documentos $/ 22 /$ informe-defensor-profesor-y-conclusiones-2016-2017 +151133934622 a.pdf

Bakker, A. B., Demerouti, E. y SanzVergel, A. I. (2014). Burnout and work engagement: The JD-R approach. Annual Review of Organizational Psychology and Organizational Behavior, 1, 389-411. DOI: 10.1146/annurevorgpsych-031413-091235

Bermejo-Toro, L., Prieto-Ursúa, M. y Hernández, V. (2016). Towards a model of teacher well-being: Personal and job resources involved in teacher burnout and engagement. Educational Psychology, 36(3), 481-501. DOI: $10.1080 / 01443410.2015 .1005006$

Durán, A., Extremera, N. y Rey, L. (2010). Analyzing the contribution of emotional intelligence and core self-evaluations as personal resources to employee engagement. En Albrecht, S. (Ed). Handbook of Employee Engagement: Perspectives, Issues, Research and Practice (209-217). Cheltenham, UK: Edward-Elgar.

Extremera, N. y Fernández-Berrocal (2015). Inteligencia emocional y educación. Madrid: Editorial Grupo 5.

Extremera, N., Mérida-López, S., SánchezÁlvarez, N. y Quintana-Orts, C. (2018). How does emotional intelligence make one feel better at work? The mediational role of work engagement. International 
Journal of Environmental Research and Public Health, 15, 1909. DOI: 10.3390/ ijerph 15091909

Extremera, N., Rey, L. y Pena, M. (2010). La docencia perjudica seriamente la salud. Análisis de los síntomas asociados al estrés docente. Boletín de Psicología, 100, 43-54.

Extremera, N., Rey, L. y Sánchez-Álvarez, N. (2019). Validation of the Spanish version of Wong Law Emotional Intelligence Scale (WLEIS-S). Psicothema, 31(1), 94-100. DOI: $10.7334 /$ psicothema2018.147

Hayes, A.F. (2013). Introduction to mediation, moderation, and conditional process analysis: A regression-based approach. New York: Guilford Press.

Instituto Nacional de Seguridad e Higiene en el Trabajo (2015). Encuesta Nacional de Gestión de Riesgos Laborales en las Empresas - ESENER 2 España. Ministerio de Empleo y Seguridad Social. Disponible en: $\quad$ http://www.oect.es/inshtweb/ contenidos/documentacion/fichas $\% 20$ de\%20publicaciones/en\%20catalogo/ observatorio/esener\%20dos.pdf

Limonero, J. T., Tomás-Sábado, J, FernándezCastro, J., Aradilla, A. Vinyes J., GómezRomero M.J, Sinclair V. G. y Wallston K. A. (2010). Adaptación española de la Brief Resilient Coping Scale: análisis preliminar. Medicina Paliativa, 17 (Supl. 1), 65.

Mansfield, C. F., Beltman, S., Broadley, T. y Weatherby-Fell, N. (2016). Building resilience in teacher education: an evidenced informed framework. Teaching and Teacher Education, 54, 77-87. DOI: 10.1016/i.tate.2015.11.016

Martínez-Monteagudo, M. C., Inglés, C. J., Granados, L., Aparisi, D. y GarcíaFernández, J. M. (2019). Trait emotional intelligence profiles, burnout, anxiety, depression, and stress in secondary education teachers. Personality and Individual Differences, 142, 53-61. DOI: 10.1016/i.paid.2019.01.036

Mayer, J. D. y Salovey, P. (1997). What is emotional intelligence? En P. Salovey y D. Sluyter (Eds.), Emotional development and emotional intelligence: Implications for educators (pp. 3-31). Nueva York, NY: Basic Books.

Mérida-López, S. y Extremera, N. (2017). Emotional Intelligence and teacher burnout: A systematic review. International Journal of Educational Research, 85, 121 130. DOI: $10.1016 /$ i.ijer.2017.07.006

Mérida-López, S., Bakker, A. B. y Extremera, N. (2019). How does emotional intelligence help teachers to stay engaged? Cross-validation of a moderated mediation model. Personality and Individual Differences, 151. https:// doi.org/10.1016/i.paid.2019.04.048

Mérida-López, S., Extremera, N. y Rey, L. (2017). Emotion-regulation ability, role stress and teachers' mental health. Occupational Medicine, 67(7), 540-545. DOI: 10.1093/occmed/kqx125

Palomera, R., Briones, E., Gómez-Linares, A. y Vera, J. (2017). Cubriendo el vacío: mejorando las competencias sociales y emocionales del profesorado en formación. Revista de Psicodidáctica, 22(2), 142-149. DOI: 10.1016/i.psicod.2017.05.002

Parker, P. D., Martin, A. J., Colmar, S. y Liem, G. A. (2012). Teachers' workplace well-being: Exploring a process model of goal orientation, coping behavior, engagement, and burnout. Teaching and Teacher Education, 28(4), 503-513. DOI: 10.1016/i.tate.2012.01.001

Richards, K., A., Hemphill, M. A. y Templin, T. J. (2018). Personal and contextual factors related to teachers' experience with stress and burnout. Teachers and Teaching, 24, 768-787. DOI: 10.1080/13540602.2018.1476337

Salanova, M., Martínez, I. M. y Llorens, S. (2005). Psicología Organizacional Positiva. En F. Palací (Ed.), Psicología de la Organización (pp. 349-376). Madrid: Pearson Prentice Hall.

Salanova, M., Schaufeli, W. B., Llorens, S., Peiró, J. M. y Grau, R. (2000). Desde el "burnout" al "engagement": ¿̇una nueva perspectiva? Revista de Psicología del Trabajo y de las Organizaciones, 16(2), $117-134$. 
Schaufeli, W.B., Salanova, M., González-Romá, V. y Bakker, A. B. (2002). The measurement of engagement and burnout: A two sample confirmatory factor analytic approach. Journal of Happiness Studies, 3(1), 71-92. DOI: 10.1023/A: 1015630930326

Schonfeld, I. S., Bianchi, R. y Luehring-jones, P. (2017). Consequences of job stress for the mental health of teachers. En T. M. Mclntyre, S. E. McIntyre y D. J. Francis (Eds.), Educator stress: An occupational health perspective, 1 (pp. 55-75). Cham, Switzerland: Springer International Publishing.

Sinclair, V. G. y Wallston, K. A. (2004). The development and psychometric evaluation of the Brief Resilient Coping Scale. Assessment, 11, 94-101. DOI: 10.1177/1073191103258144

Tait, M. (2008). Resilience as a contributor to novice teacher success, commitment, and retention. Teacher Education Quarterly, 57-75.

Taris, T. W., Leisink, P. L. M. y Schaufeli, W. B. (2017). Applying occupational health theories to educator stress: contribution of the Job Demands-Resources Model. En T. M. Mclntyre, S. E. Mclntyre y D. J. Francis (Eds.), Educator stress: An occupational health perspective, 1 (pp. 237-259). Cham, Switzerland: Springer International Publishing.

Travers, C. (2017). Current knowledge on the nature, prevalence, sources and potential impact of teacher stress. En T. M. Mclntyre, S. E. Mclntyre y D. J. Francis (Eds.), Educator stress: An occupational health perspective, 1 (pp. 237-259). Cham, Switzerland: Springer International Publishing.
Vesely, A. K., Saklofske, D. H. y Leschied, A. D. (2013). Teachers - the vital resource: The contribution of emotional intelligence to teacher efficacy and well-being. Canadian Journal of School Psychology, 28, 71-89. DOI: $10.1177 / 0829573512468855$

Vesely, A. K., Saklofske, D. H. y Nordstokke, D. W. (2014). El training and pre-service teacher wellbeing. Personality and Individual Differences, 65, 81-85. DOI: 10.1016/i.paid.2014.01.052

Watlington, E., Shockley, R., Guglielmino, P. y Felsher, R. (2010). The high cost of leaving: An analysis of the cost of teacher turnover. Journal of Educational Finance, 36(1), 22-37. DOI: 10.1353/ief.0.0028

Wheeler, A.R., Shanine, K.K., Leon, M.R. y Whitman, M.V. (2014). Student recruited samples in organizational research: A review, analysis, and guidelines for future research. Journal of Occupational and Organizational Psychology, 87, 1-26. DOI: $10.1111 /$ ioop. 12042

Wong, C.-S. y Law, K. S. (2002). The effects of leader and follower emotional intelligence on performance and attitude. The Leadership Quarterly, 13(3), 243-274. DOI: $10.1016 / 51048-9843(02) 00099-1$

Zeidner, M. y Matthews, G. (2018). Grace under pressure in educational contexts: Emotional intelligence, stress, and coping. En K. V. Keefer, J. D. A. Parker y D. H. Saklofske (Eds.), Emotional intelligence in education: Integrating research with practice, 1 (pp. 83-134). Cham, Switzerland: Springer International Publishing. 